\title{
Cosmopolitismo, constructivismo y liberalismo institucional: diálogo teórico en torno a la cooperación internacional para el desarrollo
}

\section{Cosmopolitanism, constructivism and institutional liberalism: a theoretical dialog about international development cooperation}

\section{Luis Ochoa Bilbao ${ }^{1}$}

Benemérita Universidad Autónoma de Puebla (México)

\author{
Juan Pablo Prado Lallande ${ }^{2}$ \\ Benemérita Universidad Autónoma de Puebla (México)
}

Recibido: 20-01-17

Aprobado: 20-03-17

\section{Resumen}

El artículo propone una lectura que permita vincular las teorías cosmopolita, constructivista y liberal institucional a partir de un diálogo respecto a la cooperación internacional para el desarrollo. Las tres teorías suponen una reconsideración del nuevo orden mundial que, a su vez, requiere de ajustes teóricos e institucionales para afrontar los retos de un sistema internacional más interdependiente y de alcance global. A su vez, las tres teorías resaltan la evidencia empírica que demuestra la debilidad del régimen de gobernanza global ante las resistencias de los Estados nacionales celosos de su poder y de

\footnotetext{
${ }^{1}$ (luis.ochoa@correo.buap.mx) Internacionalista y sociólogo. Profesor de la Licenciatura en Relaciones Internacionales en la Benemérita Universidad Autónoma de Puebla. Miembro del Cuerpo Académico "Política Exterior y Cooperación Internacional". Autor del libro La carrera de relaciones internacionales en México. Orígenes y situación actual, México, El Colegio de México/BUAP, 2011.

2 (juanp.prado@correo.buap.mx) Internacionalista egresado de la Universidad nacional Autónoma de México, magíster en Cooperación Internacional y doctor en Relaciones Internacionales por la Universidad Complutense de Madrid y doctor en. Profesor de la Licenciatura en Relaciones Internacionales en la Benemérita Universidad Autónoma de Puebla. Miembro del Cuerpo Académico "Política Exterior y Cooperación Internacional". Autor del libro La Cooperación Internacional para el Desarrollo. Acciones, desafios, tendencias, Madrid, La Catarata, 2015.
} 
su autoridad para dictar los lineamientos de la agenda internacional sin recurrir a esquemas conciliatorios y cooperativos.

Palabras-clave: Cosmopolitismo, Cooperación Internacional para el Desarrollo, liberalismo institucional, constructivismo.

\begin{abstract}
The article proposes the revision of cosmopolitanism, constructivism and liberal institutionalism to link them according a new and desirable dialogue on international development cooperation. The three theories intended to reconsider the new world order that requires theoretical and institutional adjustments, to face the challenges of a more interdependent and global environment. At the same time the three theories outline the evidence that shows the weakness of the global governance regime facing the resistance of the nation states, whom insists in preserve its power and authority to establish the priorities of the global agenda, outside of conciliatory and cooperative schemes.
\end{abstract}

Key-words: Cosmopolitanism, International Cooperation, Neoliberalism, Constructivism.

\title{
Introducción
}

La cooperación internacional para el desarrollo (CID) “... comprende las acciones que llevan a cabo los miembros de la comunidad internacional para apoyar, de manera solidaria, a los países que así lo requieren en sus esfuerzos por alcanzar mejores condiciones de vida para sus ciudadanos" (Figueroa, 2014: 7).

Es decir, su lógica se alimenta de una amplia gama de motivaciones que dependen de las percepciones, preferencias y objetivos de quienes practican esta actividad. A partir de la preponderancia de estas y otras aspiraciones, quienes recurren a la CID -tanto donantes como receptores encumbrados en el precepto de socios del desarrollo- despliegan un conglomerado de actividades de diversa naturaleza y alcance. Una de sus metas esenciales y aspiracionales es promover propósitos globales que trasciendan a la lógica propia del Estadonación, a efecto de generar bienes públicos con carácter sostenible que produzcan, amplíen y repartan con equidad los beneficios que se desprenden de la interdependencia y globalización. Desde esta visión, y aunque en varios casos su praxis se alinea a la política exterior de sus practicantes en donde el referente es el interés nacional del oferente, la CID se configura como un 
recurso a favor de la ciudadanía y gobernanza global, en razón a los propósitos, normas y procedimientos que emanan de su actuar.

A la luz de lo anterior, distintos enfoques teóricos de carácter multidisciplinario se dan a la tarea de aportar postulados que contribuyan a explicar los fundamentos y alcances de la CID. En este sentido, destacan tres perspectivas teóricas que resaltan el fundamento solidario de la CID contemporánea por encima de su utilización como recurso gubernamental de poder. Las tres teorías abordan el tema de manera aislada, aunque de forma concomitante explican dicho fundamento solidario de la CID: el cosmopolitismo, el constructivismo y el liberalismo institucional. Tomando en cuenta esto, el objetivo general del artículo es identificar las coincidencias entre los fundamentos más representativos del cosmopolitismo, el constructivismo y el institucional tomando como referente a la CID.

La estrategia metodológica consiste en analizar los puntos de convergencia entre las tres propuestas teóricas a efecto de corroborar si existe un "diálogo teórico" o coincidencias epistemológicas entre el cosmopolitismo, el constructivismo y el institucional para analizar a la CID contemporánea. Para ello se utilizan como marco de referencia premisas clave del cosmopolitismo, haciendo énfasis en los ocho principios cosmopolitas de David Held como formulaciones arquetípicas. De igual forma se procede respecto al constructivismo y el liberalismo institucional, con el fin de identificar los elementos de convergencia más significativos entre sí. El argumento central del artículo es que los principios del cosmopolitismo moderno coinciden en buena medida con los postulados constructivistas y liberales-institucionales, en términos de que la CID es un mecanismo internacional de perfil incluyente y democrático que pretende promover objetivos universales a favor del bienestar y sostenibilidad a escala planetaria, lo que permite aseverar que existen "vasos comunicantes" entre dichas teorías para analizar e interpretar la CID como objeto de estudio. Los vasos comunicantes concuerdan en proponer un nuevo arreglo ético en las políticas internacionales que limite la autoridad del Estado nacional, que pugne por una visión incluyente que abarque a la humanidad en su totalidad y que asumen la responsabilidad de actores gubernamentales y privados tanto en la toma de decisiones como en sus acciones. Al mismo tiempo, se argumenta que las tres teorías enfrentan resistencias fácticas por parte de los Estados nacionales cuando éstos apelan a sus intereses para promover o condicionar sus compromisos de CID o proveerla con fines egoístas que no contribuyen a una gobernanza global más democrática y consolidada. Es en esta situación en donde las tres teorías parecieran aceptar implícitamente el canon realista en lo que David Held llama "la paradoja de nuestro tiempo" (Held, 2012). 
Tras esta introducción, el artículo está compuesto de cuatro apartados y unas reflexiones finales. El primero aborda el contexto internacional que explica la perspectiva teórica del cosmopolitismo contemporáneo. El segundo detalla los ocho principios cosmopolitas descritos por Held. El tercero ofrece un panorama sintético y general de las premisas del constructivismo de la mano de los planteamientos de David. H. Lumsdaine y del liberalismo institucional con base en Keohane, Sorensen y otros referentes respecto a la CID, en donde se identifican las convergencias con respecto a los postulados cosmopolitas. Las reflexiones finales resaltan las conclusiones generales de este artículo, en el sentido de que se constata que los tres referentes teóricos comparten visiones, enfoques y explicaciones afines entre sí con respecto a la dinámica de la CID en su perfil de instrumento solidario impulsor de mayores cánones de interacción y prosperidad en la humanidad. Lo anterior permite aseverar que aunque no hay convergencia absoluta entre sí, existe un diálogo teórico por parte de las tres posturas analíticas que contribuye a explicar a la CID en su dimensión de mecanismo ético y solidario a favor de la gobernanza global y de la peldaños a favor del bienestar y sostenibilidad de la humanidad.

\section{El cosmopolitismo y la cooperación internacional para el desarrollo}

La orientación filosófica del cosmopolitismo en la teoría de las Relaciones Internacionales se enmarca en la tradición liberal y ética de la política internacional. En el mundo anglosajón el concepto de International Political Theory hace referencia al alcance internacional de la teoría política y reconoce como punto de partida el libro de Charles Beitz, Political Theory and International Relations, publicado en 1979 (Griffiths, et. al., 2009). La propuesta de Beitz consiste, fundamentalmente, en retomar los principios de la justicia propuestos por John Rawls (Theory of Justice, 1971) como baseo para una teoría política a escala global. El argumento central señala que es insostenible la idea de la soberanía Estatal en menoscabo de la libertad y el derecho a la autodeterminación de los individuos. Esta aseveración la fundamenta a partir de una idea de justicia que se sustenta en la obligación moral de las personas para ayudar a los miembros más débiles o desfavorecidos de la comunidad internacional (Rubin, 1980: 403). En este sentido se defiende la noción de justicia "como la distribución ideal y global de derechos" (Rubin, 1980: 403). A partir de aquí autores como Terry Nardin, Michael Walzer y David Held serán ubicados en el campo de la ética internacional por enfocarse en estudiar temas como la "justicia, la distribución global de recursos, la equidad, la teoría de la guerra justa, la universalidad de los derechos humanos, las libertades políticas, la paz y la responsabilidad política" (Griffiths, et. al., 2009, 309). 
El cosmopolitismo es epistemológicamente realista, deontológicamente liberal e imaginativamente universalista. Es realista en su descripción del mundo, de sus actores, de sus interacciones y de una realidad dada que es el punto de partida para una discusión sobre el cambio necesario. En este sentido, el cosmopolitismo acepta la centralidad de los Estados nacionales, comprende las estructuras de poder globales, aplaude la emergencia de una incipiente sociedad civil global y resalta la existencia de instituciones y organizaciones multilaterales. El contenido realista del cosmopolitismo es fundamental para describir el estatus quo del poder mundial e incidir en las posibilidades de su transformación.

Precisamente en su afán por el cambio, el cosmopolitismo es liberal en cuanto a la promoción de los valores cívicos, democráticos e incluyentes enmarcados en los preceptos éticos occidentales. Sin embargo, la teoría cosmopolita de las Relaciones Internacionales que abordamos en este trabajo no es la del canon idealista cuya tradición encuentra en Kant a uno de sus más importantes referentes ${ }^{3}$. Más que la discusión sobre la universalidad de la condición humana y los requisitos para aceptarla y protegerla, más allá del ideal cívico de hacer de "cada rincón del mundo el hogar de todo ser humano", la perspectiva cosmopolita que nos interesa en este trabajo tiene un perfil más pragmático. Como escribe Carla Millán, el cosmopolitismo "parece ser una doctrina adecuada para entender y gestionar la nueva realidad social de interdependencia e interconexión de los fenómenos políticos, sociales, económicos y medioambientales" (Millán, 2014: 174). Este es el sentido pragmático del cosmopolitismo de David Held, ya que se trata de una mirada que reconoce las estructuras dadas del poder global, que no desprecia a los actores clásicos ni a los emergentes del sistema internacional, pero que a la vez tampoco desconoce que en el mundo "existe una densa red de acciones económicas y políticas -junto con sus multidimensionales consecuencias- que no reconocen los límites de las fronteras" (Millán, 2014: 174). Y aquí resulta muy importante señalar que esta transformación del sistema internacional no es necesariamente consciente; es decir, no responde a una planificación racional ni a una elección voluntaria (Beck, 2005; Millán, 2014: 174). Simplemente la interdependencia y transnacionalización del acontecer mundial son fenómenos que están ocurriendo y que parecieran estar adquiriendo un perfil liberal e institucional.

En este punto el cosmopolitismo es "imaginativo" (Kendall, Woodward \& Skrbis, 2009: 36), porque asume los cambios estructurales del sistema internacional como una condición contemporánea y dado a que pretende

\footnotetext{
${ }^{3}$ Kant ha sido recuperado en diversos trabajos contemporáneos de las Relaciones Internacionales para rescatar sus aportaciones al neoliberalismo moderno, el cosmopolitismo y el neoisntitucionalismo. Véanse: Linklater \& Hidemi, 2006; Beate, 2006; Sutch \& Elias, 2007; Griffiths, et. al., 2009.
} 
incidir en dichos cambios para orientarlos hacia la consolidación de los valores liberales e institucionales que defienden los teóricos cosmopolitas. Es, por tanto, una perspectiva teórica que propone transformar el orden social existente (Kendall, Woodward \& Skrbis, 2009, p. 36) otorgándole a dicha empresa un carácter universal, que se conecta con la concepción elemental liberal de la condición humana.

Las reflexiones teóricas contemporáneas sobre el cosmopolitismo se plantean la necesidad de proponer un nuevo arreglo ético político ante las transformaciones del sistema internacional. Desde esta óptica la globalización se considera irreversible y eso trae consigo la inevitable erosión del concepto de soberanía estatal. Quizá este sea el punto de partida de los cosmopolitas: el hecho de que los Estados nacionales no son autosuficientes y tampoco son autónomos en sus acciones de política externa e interna. Más aun, los Estados nacionales tampoco son ya la única fuente de autoridad legítima en un mundo más diverso y complejo, con nuevos arreglos institucionales y nuevos actores incorporados al universo de toma de decisiones (Czempiel and Rosenau, 1992; Leibfried and Zürn, 2005).

Aquí se propone un cambio epistemológico para transitar del concepto de gobierno al concepto de gobernanza (Pierre, 2000; Sand, 2004; Slaughter, 2004). En consonancia con el cosmopolitismo, la gobernanza se plantea desde "el contexto de la globalización", ampliar el marco para la comprensión de la autoridad más allá del Estado nacional, resalta la diversidad de relaciones y de actores en el espacio internacional, y dentro de ese marco de diversidad los actores internacionales tienden a "homologar sus políticas hacia agendas comunes" en un mapa complejo caracterizado por "múltiples canales de interacción" en una estructura global que "cambia constantemente" (LópezVallejo, 2016: 474-475).

Lo anterior reitera la noción de que el mundo se ha vuelto interdependiente. Y no sólo en el terreno financiero o económico. Los cosmopolitas alertan sobre el enorme abanico de interacciones sociales y culturales tan inevitables como la globalización y la propia cooperación internacional, que obligan a buscar nuevas vías de entendimientos básicos y que no pueden ser los mismos del “interés nacional", la razón de Estado, el etnonacionalismo, la autarquía, el aislacionismo o la excepcionalidad. Es comprensible que los cosmopolitas dirijan sus argumentos contra las interpretaciones clásicas más rígidas del realismo en las Relaciones Internacionales y promuevan conceptos nuevos como "realismo imaginativo" (Kendall, Woodward \& Skrbis, 2009, p. 5) o "realismo cosmopolita" (Beck, 2006). En resumen, el arreglo es el siguiente: el cosmopolitismo tiene una base realista internacionalista porque asume la persistencia del poder estado céntrico y una base filosófica realista ante la necesidad de contar con instituciones que regulen el comportamiento egoísta 
humano y de los Estados. En suma, no niegan, sino que buscan redirigir hacia el ideal cosmopolita, el comportamiento del Estado revalorando, al mismo tiempo, la importancia de la esfera pública y de la ley (Kendall, Woodward \& Skrbis, 2009, p. 5).

Es, además, una declaración de principios ya que los cosmopolitas promueven nuevos arreglos éticos, y la ética y la moral han sido históricamente marginalizados por los discursos realistas y neorrealistas de la política internacional. Y el nuevo arreglo ético propuesto por los cosmopolitas de alguna forma apunta a que todas las acciones de los otrora poderosos, autónomos y soberanos Estados nacionales, tienen repercusiones a gran escala y que involucran a la humanidad (Beck, 2006). Por lo tanto, la interdependencia no sólo justificaría el giro ético de la política internacional por la naturaleza de las relaciones económico financieras entre los países, sino porque las consecuencias de toda acción estatal son ahora, más que nunca, consecuencias globales.

Los cosmopolitas avizoran un tenue entendimiento de tal realidad por parte de las élites gobernantes, en la construcción y reconstrucción de las relaciones transnacionales que profundizan los niveles de información compartida por las Estados nacionales en organizaciones colectivas ya sea de alcance regional o global. Hasta la primera década del siglo XXI, por ejemplo, se señalaba que uno de los más grandes éxitos de la Unión Europea (UE) había sido el sistema de información transnacional con el que los Estados miembros, mediante diversos ejercicios cooperativos comparten datos y cifras sobre la adquisición de armas o la innovación tecnológica militar (Cooper, 2003). Lo que antes era un secreto por razones estratégicas de seguridad se convirtió en información compartida también por razones de seguridad, pero con alcances más amplios y nuevos horizontes de responsabilidad. Si bien la lógica de la amenaza externa no se desmanteló por completo, la UE logró reconstruir el espacio interno compuesto por Estados nacionales que ya no serían más una amenaza entre sí, sino los aliados más confiables para cooperar en rubros cada vez más amplios y ambiciosos. Para los cosmopolitas este ejercicio transnacional había sido emblemático de los potenciales éxitos de los nuevos arreglos éticos de la convivencia entre las naciones.

Una primera conclusión hasta aquí resalta que la globalización, y la construcción de redes transnacionales y en definitiva la cooperación internacional ocurren en el mundo interconectado contemporáneo y plantean la necesidad de alcanzar un nuevo arreglo ético para la política y el orden internacional. El cosmopolitismo se convierte así en una respuesta de carácter ético ante el hecho social que significa la globalización (Kendall, Woodward \& Skrbis, 2009, 149). Ésta, de carácter irreversible, debe ser entendida en su primera fase como la paulatina erosión de las fronteras tradicionales del sistema internacional. Esto implica el rediseño de los alcances del Estado nacional, 
especialmente por la emergencia de nuevos actores internacionales quienes mediante ejercicios cooperativos promueven la conquista de aspiraciones universalistas como el desarrollo, la justicia, la igualdad, la equidad o justicia distributiva, los derechos humanos y la sostenibilidad de medio ambiente. El cosmopolitismo es, por lo tanto "un proyecto intelectual y político que hace la promesa de una sociedad civil global" $y$, "basados en el principio de solidaridad" le pedirían a la comunidad internacional (a la humanidad) "reconsiderar sus lealtades locales como la base primaria de la interacción social y cultural" (Kendall, Woodward \& Skrbis, 2009, 149). En un mundo globalizándose, interdependiente y paulatinamente transnacional, la realidad ya no puede sostenerse de manera particularista, localista o parroquial. Este clamor es, todavía, como se ha indicado, un proyecto intelectual.

Finalmente, cabe decir que el cosmopolitismo propone una nueva ontología de las relaciones internacionales, y como tal, es también un proyecto transgresor. El giro ético de la política internacional necesariamente pasaría por la contención arbitraria del poder de los Estados nacionales y por el rediseño o la cancelación de las instituciones mundiales heredadas del siglo XX y aparentemente obsoletas en la actualidad como la ONU, el Fondo Monetario Internacional, el Banco Mundial y la Corte Internacional de Justicia.

\section{David Held y los ocho principios del cosmopolitismo moderno}

La obra de David Held es, en un primer momento, un intento por sintetizar los puntos de acuerdo entre el liberalismo y el marxismo, teniendo siempre al modelo democrático como piedra angular de dicha síntesis. La democracia es fundamental en su trabajo, tanto es así que discute los riesgos de la democracia ante la era de la globalización. Por supuesto, también avizora que la globalización puede ser benéfica para la democracia considerándola como "un bien público" que logre expandirse por el orbe y que pueda garantizar la igualdad, la justicia y la libertad (Griffiths, et. al., 2009: 318-326). Para el mundo académico, Held es considerado el promotor de la "democracia cosmopolita" más relevante. Al distinguir tres tipos de cosmopolitismo: el político, el legal y el liberal, pretende diseñar las "obligaciones, las prerrogativas institucionales, los derechos morales y los factores ético-políticos que han propiciado un nuevo orden mundial constitucional" (Griffiths, et. al., 2002: 57).

Held propone una "democracia cosmopolita" que haga patente ese tránsito contemporáneo para superar las fronteras artificiales de los Estados y que se evidencia en la formalización institucional de múltiples arreglos legales entre los Estados nacionales, independientemente de que sean relaciones bilaterales o multilaterales. Pero, consciente de las críticas que se desprenderían de una noción eurocéntrica de los valores éticos (individuo, libertad, derechos 
universales, propiedad privada), Held plantea un proyecto cosmopolita contemporáneo que reconozca la variabilidad cultural al mismo tiempo que la sensatez de los arreglos mínimos indispensables entre dos o más entidades para que la cooperación tenga éxito:

[el proyecto cosmopolita moderno] se trata es de un ejercicio más concreto, que tiene como objetivo reflexionar sobre el estatus moral de las personas, las condiciones de acción y la toma colectiva de decisiones. Es importante hacer hincapié en el hecho de que este ejercicio se construye a partir de la aceptación de las normas básicas de la comunicación, el diálogo y la solución de controversias, que no son sólo deseables, sino también esenciales precisamente porque todas las personas tienen el mismo valor moral y sus opiniones en torno a un gran número de cuestiones político-morales pueden entrar en conflicto. Los principios del cosmopolitismo son condiciones que toman en serio la diversidad cultural y la construcción de una cultura democrática para mediar en los conflictos sobre el bien cultural. En resumen, tratan sobre condiciones de diferencias justas y diálogo democrático. El objetivo del cosmopolitismo moderno en la conceptuación y la generación de las condiciones necesarias para una estructura "básica" o "común" de acción individual y actividad social" (Held, 2005, 139).

En este sentido, el cosmopolitismo no sería un "marco fundamental de referencias que se imponga a todas las demás posiciones morales"; más bien, se trataría de "un subconjunto determinado de consideraciones que establece que existen algunas reglas universales fundamentales, normas y principios de justicia que deberían sopesarse y tenerse en cuenta ante las derivadas de sociedades concretas y otros grupos humanos" (Held, 2005, 141).

Las propuestas de Held, tanto de una democracia cosmopolita global como la de los ocho principios cosmopolitas, surgen de una mirada descriptiva y normativa respecto al funcionamiento de la política internacional. El enfoque normativo de Held debe considerarse como un recetario de las "buenas prácticas" que ya han reportado éxitos en el sistema internacional y que tendrían que replicarse de manera ordenada.

Held advierte que su concepto de cosmopolitismo "trata de revelar la base jurídica, cultural y ética del orden político en un mundo en el que las comunidades políticas y los estados importan mucho, pero no única $\mathrm{y}$ exclusivamente" (Held, 2005: 133). A partir de una reconfiguración del poder internacional, como se ha indicado antes, con nuevos actores, con relaciones de cooperación e interdependencia y con trascendencia más allá de las fronteras físicas y simbólicas de los Estados nacionales, Held distingue los ocho principios cosmopolitas que le otorgarían una dimensión humana a la política internacional. Dichos principios se citan textualmente a continuación (Held, 2005: 134-138): 
1) "igual valor y dignidad: El primer principio señala que las unidades primordiales de la preocupación moral son los seres humanos, y no los estados u otras formas de asociación humana. La humanidad pertenece a un único reino moral en el que cada persona es igualmente merecedora de respeto y consideración";

2) "participación activa; El segundo principio reconoce que, si el primero se admite y acepta universalmente, la acción humana no puede, entonces, entenderse como una mera expresión fruto de una teleología, una fortuna o una tradición dada; más bien, la acción humana se debe concebir como la capacidad para actuar de forma diferente - la capacidad no sólo de aceptar sino también de conformar la comunidad humana en el contexto de las elecciones de los demás";

3) "responsabilidad personal y pública (accountability): Los principios 1 y 2 no se pueden entender por completo a menos que se complementen con el principio tercero: el principio de la responsabilidad personal y pública [...] Los actores deben ser conscientes, y responsables, de las consecuencias de sus acciones, directas o indirectas, intencionadas o no, que pueden restringir o limitar radicalmente las elecciones de los demás. Los individuos tienen tanto derechos, cuanto obligaciones en lo relativo a su responsabilidad personal";

4) "consentimiento; igual valía y del igual valor moral, junto con la participación activa y la responsabilidad personal, exigen un proceso político no coercitivo por medio del cual los individuos puedan negociar y llevar a cabo sus interconexiones e interdependencias públicas, así como las oportunidades que se les presenten en su vida [...] El principio del consentimiento constituye la base del acuerdo colectivo no coercitivo y de la gobernabilidad";

5) "toma de decisiones colectiva mediante procedimientos democráticos en lo referente a los asuntos públicos: El principio 5 reconoce la importancia de la inclusividad a la hora de dar el consentimiento, de lo que se deduce que un proceso inclusivo de participación y debate puede ir de la mano de un proceso de toma de decisiones que conduzca a unos resultados que aglutinen el mayor apoyo posible";

6) "inclusividad y subsidiariedad: El sexto principio, (...) pretende aclarar cuáles son los criterios fundamentales para establecer los límites adecuados en torno a las unidades que intervienen en la toma colectiva de decisiones [...] De acuerdo con el sexto principio, la toma colectiva de decisiones es más útil cuanto más cercana y más implica a aquellos cuyas perspectivas de vida y oportunidades está determinadas por importantes fuerzas y procesos sociales";

7) "evitar daños graves: El séptimo principio es el de la justicia social; un principio que consiste en evitar el daño y mejorar las necesidades más apremiantes. Este principio pretende dar prioridad a los casos de necesidad más vital y, siempre que sea posible, pasar por alto otras prioridades menos urgentes hasta que todos los seres humanos tengan garantizados, de facto y de jure, los seis primeros principios"; 
8) "sostenibilidad: El octavo y último principio es el de la sostenibilidad, que precisa que todo desarrollo económico y social debe procurar la buena administración de los recursos básicos del mundo - por "básicos" me refiero a aquellos recursos que son irreemplazables e insustituibles. Este principio excluye cualquier cambio económico y social que perturbe el equilibrio ecológico mundial, y que dañe innecesariamente las oportunidades de las generaciones futuras. El desarrollo sostenible ha de entenderse como un principio orientador, y no como una fórmula exacta, ya que no sabemos, por ejemplo, cómo podrán influir las futuras innovaciones tecnológicas en el uso y suministro de los recursos".

A su vez, los principios aquí expuestos deben ser entendidos: 1) en un nuevo contexto internacional; 2) como una forma de acción política y; 3 ) como un ideal que enfrenta un obstáculo en particular.

1) El nuevo contexto internacional, dice Held, no debe supeditarse a una concepción territorial. Por el contrario, la "autodeterminación, la responsabilidad, la democracia y la soberanía" tendrán que interpretarse tomando en consideración "múltiples formas de afiliación: local, nacional y global. Los principios cosmopolitas son el elemento básico de la vida pública democrática, despojado del vínculo contingente con las fronteras de los Estados-nación" (Held \& Pattomaki, 2006, 92).

2) El cosmopolitismo como acción política lo explica Held a partir de su trabajo y sus experiencias en el Network Institute for Global Democratization (NIGD) que se trata de una "ONG transnacional con sede en Helsinki y especie de colectivo intelectual orgánico". Ahí, dice Held, "hemos desarrollado un proyecto para estudiar diversas iniciativas de democracia global, con una intencionalidad política y práctica (el proyecto fue financiado y respaldado por el Departamento de Cooperación para el Desarrollo del Ministerio de Asuntos Exteriores finlandés). Diversos debates celebrados en otoño de 2000 desembocaron en el desarrollo de un plan en tres fases para un diálogo Norte-Sur sobre la democratización global. Los tres pasos previstos eran: 1) una sesión de lluvia de ideas sobre iniciativas de democracia global; 2) una evaluación sistemática de estas iniciativas; y 3) una gran conferencia inter y transnacional donde se elegirían la mejor o las mejores iniciativas para la acción concreta, con la posibilidad de formalizar la conclusión por medio de un tratado o carta internacional. En el curso de las dos primeras fases, fue evidente que, en lugar de seleccionar iniciativas sin más, hace falta crear lazos entre diferentes reformas y desarrollar una estrategia sistemática y holística sobre esa base" (Held \& Pattomaki, 2006, 97). 
3) Finalmente, Held identifica un obstáculo para los alcances del proyecto cosmopolita contemporáneo que él ha denominado la paradoja de nuestro tiempo: "los problemas colectivos a los que nos enfrentamos son cada vez más globales $\mathrm{y}$, sin embargo, los medios de que disponemos para abordarlos son nacionales o locales, débiles e incompletos" (Held, 2012: 139). Por un lado, Held destaca patrones históricos y clásicos de distribución de poder que enfrentan cambios estructurales como los nuevos "mecanismos de coordinación y cooperación multilaterales", sin embargo, ante situaciones de crisis como la de seguridad en 2001 o la económica en 2008, "cuando lo que se requiere es una estrategia mundial, el poder está organizado ampliamente sobre una base nacional (Held, \& Young, 2011: 624).

El abordaje sobre el cosmopolitismo hecho hasta aquí no toca el tema de la CID de manera central. Sin embargo, las reflexiones sobre la erosión del poder del Estado nacional, la globalización y la interdependencia, la necesidad de nuevos arreglos éticos y democráticos, así como el aparente ascenso de una sociedad civil global, demuestra un conglomerado de ideas y supuestos que los estudiosos de las CID tienen como eje primordial. En otras palabras, el cosmopolitismo, sin hablar directamente de la CID, hace referencia a todos los arreglos institucionales necesarios para la consolidación de lo que se denomina sociedad internacional ${ }^{4}$. Podría decirse que el lenguaje teórico del cosmopolitismo coincide plenamente con el lenguaje teórico de la CID. En el siguiente apartado se resaltan los vasos comunicantes de lo que proponemos como el diálogo implícito cosmopolitismo-constructivismo-liberalismo institucional respecto a la CID.

\section{La cooperación internacional para el desarrollo vista desde el constructivismo y el liberalismo institucional de las Relaciones Internacionales}

La propuesta de resaltar los vínculos entre el cosmopolitismo, el constructivismo y el liberalismo institucional al momento de abordar a la CID se sostiene en el planteamiento de Markus Kornprobst (2009) quien señala la necesidad de construir un diálogo en la disciplina de las Relaciones Internacionales en la que se traslapan los horizontes en lugar de contener

${ }^{4}$ La sociedad internacional (International Society) es un concepto fundamentalmente británico vinculado a la Escuela Inglesa de las Relaciones Internacionales. Al hablar de sociedad, esta escuela enfatiza que el sistema internacional (un concepto que pretende ser neutral) en realidad actúa sobre bases reconocibles de diálogo, acuerdos y colaboración que hacen posible el funcionamiento del mundo conformado por unidades aceptadas y reconocibles como los Estados nacionales. Si bien no hay una entidad supranacional que regule y sancione a los actores, el sólo hecho de la convivencia internacional supone ya un comportamiento social y no anárquico como supondría el realismo. 
paradigmas inconmensurables. En esta idea resuenan los ecos de "abrir la Ciencias Sociales" propuestos en la década de los noventa por Immanuel Wallerstein o para tratar de salvar el peligroso destino de irrelevancia de la disciplina mediante el concepto de gobernanza global como proponen Thomas Weiss y Rorden Wilkinson (2014). Con Wallertein (2007: 101) se planteaba la necesidad de reconstruir las Ciencias Sociales para hacerlas "verdaderamente pluralistas y universales", y con Weiss y Wilkinson se argumentaba "superar la fragmentación y atomización que hoy dominan a las Relaciones Internacionales" (2014: 77) $)^{5}$.

Por su parte Kornprobst propone reconocer los elementos retóricos (en el sentido aristotélico) tanto de las perspectivas positivistas (cosmopolitismo y liberalismo institucional) como las post-positivistas (constructivismo), que en el fondo procuran una epistemología de la política internacional mucho más coincidente y complementaria de lo que sus respectivos defensores imaginarían. Se trataría de una apuesta por superar las fronteras autoimpuestas de las ortodoxias epistemológicas. En este sentido se analizan a continuación los ejes de lo que consideramos "vasos comunicantes" entre el constructivismo y el liberalismo institucional cuando abordan el tema de la CID, y que se conectan con gran naturalidad con el cosmopolitismo.

\section{El constructivismo y la cooperación internacional para el desarrollo}

Entre las teorías de las Relaciones Internacionales que analizan a la CID destaca el constructivismo; postulado post-positivista que hace énfasis en los fundamentos morales que le dan vida a esta actividad. En términos generales, los constructivistas parten de la idea de que las estructuras fundamentales de la política internacional son básicamente sociales, y no exclusivamente materiales. Estas estructuras sociales, al influir en las percepciones de los gobernantes sobre la "realidad" internacional, condicionan los intereses, los valores, la ideología y las percepciones de los actores internacionales.

Eso significa que los intereses nacionales de los países son producto de las construcciones sobre lo que los estadistas perciben respecto al contexto internacional. Estas estructuras sociales consisten en comprensiones, expectativas y conocimientos compartidos que, en su conjunto, generan las características y naturaleza de las relaciones entre los actores del sistema internacional, ya sean éstas de cooperación o de conflicto. En este sentido, igual que los cosmopolitas, los constructivistas sostienen que el elemento

\footnotetext{
${ }^{5}$ No es el objetivo de este artículo profundizar en las agendas propuestas por los autores, pero destaca el hecho de que Wallerstein, Weiss y Wilkinson así como Kornprobst insistan en fortalecer el diálogo entre estudiosos de las Ciencias Sociales y los temas internacionales, un diálogo abierto, incluyente y que supere el juego de sordos que parece existir, al menos, entre los académicos internacionalistas.
} 
moral en las relaciones internacionales tiene un importante lugar en las estructuras sociales. Los defensores de este planteamiento teórico intentan mostrar la manera en que las estructuras sociales de un sistema permiten a sus actores realizar acciones mediante una serie de valores e intereses, a través de determinados medios y capacidades (Wendt, 1995:76). Desde esta perspectiva, debido a que las relaciones internacionales, y por consiguiente, las estructuras sociales no corresponden de manera exclusiva a simples intereses nacionales de carácter egoísta y en competencia mediante una constante lucha por el poder, otros elementos como las consideraciones morales pueden explicar con mayor eficiencia la naturaleza y leitmotiv de dichas estructuras.

Lo anterior implica que más que enfocarse en estructuras políticasadministrativas sólidas como los Estados o los gobiernos, los constructivistas se concentran en las ideas y las normas que moldean las percepciones y comportamientos de los actores internacionales. Esto induce a que, desde la óptica de Wendt "el constructivismo presta gran atención a la construcción de las identidades (principios y valores compartidos, percepciones del mundo, marcos histórico-culturales generadores de identidades, mecanismos de interiorización)" (Barbé, 2007:93) [...] lo cuales inciden en las preferencias de los actores las cuales (a diferencia respecto a lo que dicta el racionalismo) no vienen dadas exógenamente, sino como producto del entorno social y de normas comunes (Barbé, 2007:93).

Por ello, "Dado el interés del constructivismo por las estructuras sociales y normativas, no es extraño que en su agenda de investigación ocupe un lugar destacado el estudio de las instituciones y de los regímenes internacionales" y por ende los Estados y gobiernos pasen a un espacio secundario (Barbé, 2007:93).

De ahí que desde este prisma teórico el constructivismo pretende construir "puentes" entre las tradiciones positivistas y post-positivistas. Lo dicho en el sentido de posicionar en los procesos explicativos aquellos factores de los que más que el comportamiento "racional" de los gobiernos per se, se ponga sobre la mesa las causantes de los mismos, los cuales a su vez dependen de normas e instituciones de los cuales resultan ejercicios concretos (racionales o no) tanto de gobiernos, como de una gama más amplia de actores internacionales.

En cuanto al estudio de la CID, el constructivismo parte de la premisa relativa a que existen sólidos fundamentos morales para que los Estados poderosos y, por supuesto, otros actores de la sociedad internacional suministren CID a terceros países y beneficiarios. Esta teoría defiende que durante décadas las consideraciones morales proporcionan un importante sustento a las políticas y programas de cooperación al desarrollo, significando ello que esta actividad (a diferencia por ejemplo del realismo) no constituye un fin en sí mismo, sino que mantiene intereses desarrollistas y solidarios donde se destina. Por tanto, 
para los constructivistas existe una amplia gama de circunstancias que generan el marco moral necesario para que los Estados fuertes promuevan desarrollo en los débiles:

1. Necesidades de las personas inmersas en pobreza en los países pobres;

2. Amplia inequidad de recursos y oportunidades entre ricos y pobres; e

3. Injustas relaciones históricas entre países desarrollados y en desarrollo, las cuales pueden ser restituidas o compensadas.

Así, para esta corriente de análisis, basta con aceptar tan sólo una de estas justificaciones para sustentar la naturaleza de la obligación moral para cooperar (Riddel, 1987: 12). Este planeamiento teórico -al igual que le sucede al cosmopolitismo, al neoliberalismo y a otras matrices teóricas- es objeto de innumerables críticas por parte de varios estudiosos de las Relaciones Internacionales (especialmente de los realistas), quienes afirman que los Estados no tienen compromisos morales extraterritoriales $\mathrm{y}$, en consecuencia, la ayuda es sólo un mecanismo más de sus políticas exteriores, cuya finalidad consiste en promover su propio interés nacional ${ }^{6}$.

David Lumsdaine, un connotado constructivista que orienta su análisis a la CID, parte del fundamento relativo a que la colaboración externa no puede explicarse únicamente con base en los intereses políticos y económicos de las principales potencias; esto es el realismo. Para este autor los elementos humanitarios y solidarios en el sistema internacional de cooperación al desarrollo han jugado, desde el inicio de su implementación, un papel fundamental; aseveración sustentada mediante una amplia e interesante gama de fundamentos teóricos, estadísticos y conceptuales.

Lumsdaine (1992:4), en coincidencia con la postura cosmopolita, considera que el interés nacional no puede explicar de manera "absoluta" el comportamiento de los Estados en el entorno internacional y, por consiguiente, tampoco lo consigue con respecto a las razones de los gobiernos donantes para suministrar ayuda externa (1992: 4). Para este investigador las concepciones morales que inciden en la política internacional consisten en: 1) La transferencia sistemática hacia lo internacional de las concepciones internas sobre los valores de justicia y desarrollo; 2) El diálogo social y moral que constituye a la sociedad internacional; y 3) El conjunto de significados normativos implícitos en los regímenes internacionales y sus respectivas actividades que emanan de éstos,

\footnotetext{
${ }^{6}$ Por ejemplo, la postura realista considera que la CID debe ser diseñada fundamentalmente, si no de forma exclusiva, para defender los intereses del donante en el Estado receptor, los cuales consisten en incrementar su influencia política, su seguridad militar, el comercio y sus respectivas inversiones externas. De esta forma, la ayuda es interpretada por dicha línea teórica como un asunto inseparable del poder (Hook, 1995: 34).
} 
tales como la cooperación internacional, los cuales le dan forma a la evolución y perspectiva de su práctica (Lumsdaine, 1992: 5).

De esta manera, para Lumsdaine (1992; 6-7), y de nueva cuenta en línea con cosmopolitas como Held, el estudio de los acontecimientos internacionales no se satisface mediante el limitado análisis realista, ya que "la naturaleza humana es mucho más compleja: el interés propio, lo irracional, la destrucción y los principios de compasión juegan, todos ellos, un papel en la política internacional, así como en la sociedad civil y en las políticas nacionales" (Lumsdaine, 1992: 6-7). Es decir, la humanidad es una mezcla de auto interés, idealismo y destrucción sin sentido alguno, operando estos tres elementos simultáneamente en la dimensión personal, en la sociedad civil, así como en el ámbito internacional (1992: 9). Es en este punto donde Lumsdaine sustenta su postura al señalar que las sociedades desarrolladas "trasladan" los comportamientos "humanitarios" suscitados ad interim de sus respectivas sociedades hacia el contexto externo mediante ayuda al desarrollo; óptica que se alinea a lo señalado por Kendall, Woodward \& Skrbis, quienes como se vio previamente también sostienen la existencia $-\mathrm{y}$ activismo- de una sociedad civil global.

Lumsdaine señala que existen tres motivos generales por lo que diversos elementos de carácter moral se encuentran presentes en las relaciones internacionales: 1) El primero es el ya referido traslado de los valores humanos nacionales hacia el ámbito externo. Esto significa que los países con sistemas internos de bienestar social consolidados se caracterizan por constituirse como importantes donantes de cooperación. 2) El segundo es que un país tiende a ser influenciado en sus instituciones nacionales debido a sus relaciones y roles que tiene con la sociedad internacional, ya que "a los Estados les interesa cómo son vistos por los otros Estados". De esta forma, "la ayuda externa se vuelve parte de la vida interna de los Estados". 3) El tercero se refiere a la influencia de los regímenes internacionales, los cuales al incidir en las relaciones del resto de los actores internacionales y, ya que éstos se encuentran siempre en constante evolución, propicia que los elementos morales ocupen espacios de cada vez mayor importancia. Por ejemplo, desde esta plataforma teórica, el hecho que los objetivos e instrumentos de la cooperación establezcan al combate a la pobreza como su premisa fundamental incita a los cooperantes a asumir este tópico como una meta e ideología de cada vez mayor relevancia. (Lumsdaine, 1992: 22-23-27).

Al respecto, Lumsdaine asevera que las relaciones entre los actores del sistema internacional contienen una importante gama de elementos de carácter moral distintos, o al menos, que complementan a los relativos a la búsqueda del interés nacional mediante meras relaciones de poder. De esta forma, Lumsdaine concluye que "la ayuda externa no puede ser explicada solamente (las cursivas 
de los autores) con base en los intereses económicos y políticos de los donantes, por lo que cualquier explicación satisfactoria al respecto debe otorgar un sitio central a la influencia de las convicciones humanitarias e igualitarias en los donantes de ayuda" (1992:29).

\section{El liberalismo institucional y la cooperación internacional para el desarrollo}

Por su parte, el liberalismo institucional, corriente teórica internacionalista particularmente útil para el estudio de determinados segmentos de la CID, también cosecha en su acervo varios postulados que convergen con respecto al cosmopolitismo y el constructivismo.

Desde esta visión, y (como lo hacen cosmopolitismo y constructivismo), partiendo del análisis de las personas en lo individual, si bien éstas son esencialmente competitivas entre sí, los liberales argumentan que los individuos, las sociedades y por ende países, al compartir intereses comunes en múltiples aspectos, son proclives a colaborar unos con otros, lo cual puede generar beneficios entre las partes involucradas. Es decir, los liberales argumentan que mediante el uso de la razón es posible realizar cooperación entre individuos; práctica que resulta plausible "elevarla" a sociedades y tras ello replicarla en ejercicios colaborativos entre países y otros protagonistas de la sociedad internacional. Como es evidente este postulado coincide con lo señalado por los cosmopolitas y constructivistas, quienes parten también del mismo planteamiento para explicar los fundamentos que le dan vida a la colaboración local, nacional e internacional. Lo anterior es posible desde la perspectiva liberal de las Relaciones Internacionales, debido a que los procesos de modernización conminan a los actores involucrados a que el interés colectivo prevalezca respecto a actitudes egoístas o conflictivas, pues de esta forma es factible generar y repartir los dividendos generados.

Como señalan Zacher y Matthew, la colaboración prevalece con respecto al conflicto, debido a que los procesos de modernización (inherentes a la creciente interrelación entre diversos sujetos, sociedades, países y regiones) incrementan los incentivos para mantener o incrementar acciones (en Jackson, Robert y Sorensen, Georg, 2010: 97).

Una rama del liberalismo es el de perfil "interdependiente". Desde esta postura, y de nueva cuenta en convergencia con respecto a lo señalado por Beck y Millán (2014), se asevera que la creciente interacción entre actores internacionales conlleva a que se generen lazos lo suficientemente fuertes y profundos que incentiven dependencia mutual entre sí. En estas circunstancias, entre mayor acercamiento y contactos entre dos o más países (y, por supuesto, actores subnacionales, públicos o privados en su seno según cada caso) en múltiples aspectos, aumentará la interdependencia, y por ende colaboración, entre ellos. 
En cuanto al liberalismo institucional, como se señaló previamente (Prado, 2016b) esta óptica teórica considera que las instituciones promueven, mejoran y aumentan la permanencia a través del tiempo de la cooperación internacional (Krasner, 1983, Keohane, 1984; Young, 1989), y de ahí el adjetivo "institucional" ". Dicho de otra forma, las instituciones son mecanismos normativos de las Relaciones Internacionales que pretenden influir en la conducta de sus actores. Lo anterior con miras a que quienes participan en su ejercicio comiencen, mantengan o incrementen sus lazos de cooperación unos con otros, consiguiendo tras ello atender necesidades comunes, previamente percibidas entre las partes involucradas. En este punto confluyen los liberales institucionales con el cosmopolitismo. De los principios cosmopolitas de David Held, la posibilidad de un trabajo coordinado y efectivo entre actores internacionales requiere del consentimiento para la toma de decisiones colectivas que supone "un proceso inclusivo de participación y debate [que] puede ir de la mano de un proceso de toma de decisiones que conduzca a unos resultados que aglutinen el mayor apoyo posible" (Held, 2005: 136-137).

El institucionalismo explora la manera en que las referidas entidades multilaterales, regionales, bilaterales, triangulares o nacionales dedicadas a la cooperación internacional (en una palabra, instituciones) interactúan unas con otras con miras a conseguir múltiples propósitos, analizando sus normas internas, estructura, procesos de toma de decisiones, instancias de coordinación, gestión interna y, por supuesto, su forma de operar y resultados generados. Éste énfasis se explica dado a que el institucionalismo asevera que la estructura, el diseño y funcionalidad de las Agencias de Cooperación Internacional, organismos multilaterales, mecanismos de cooperación, etc. juegan un rol trascendental, puesto que tales factores determinan la capacidad de estos entes para conseguir sus objetivos (Karns y Mingts, 2010:38).

Desde esta postura el funcionamiento y operatividad de las instancias dedicadas a la cooperación internacional se sustentan en la aplicación de reglas o normas. El fin de tales ordenamientos consiste en establecer procesos institucionalizados de sus actividades, en el sentido de que sus acciones y resultados sean predeterminados y durables. Para que esto sea posible, resuena como requisito el tercer principio cosmopolita de David Held: responsabilidad personal y pública o accountability. Sólo dentro en un marco normativo se pueden canalizar las habilidades y talentos diferentes, las necesidades, las aspiraciones y los intereses distintos, en un ordenamiento a partir de las prioridades y respetando la elección voluntaria, para la conquista de metas comunes. Los resultados perdurables de las acciones individuales canalizadas por las instituciones consisten, precisamente, en la permanencia de las reglas

7 A esta teoría se le conoce también como institucional y en ocasiones rational choice institutionalism.

Araucaria. Revista Iberoamericana de Filosofía, Política y Humanidades, año 19, n 37. Primer semestre de 2017. Pp. 273-299. ISSN 1575-6823 e-ISSN 2340-2199 doi: 10.12795/araucaria.2017.i37.14 
para llegar a acuerdos, y la conciencia de los actores respecto a la responsabilidad que recae en ellos por sus decisiones.

En síntesis, tanto el cosmopolitismo y el liberalismo institucional plantean que los aparatos burocráticos-administrativos dedicados a la cooperación internacional, los regímenes internacionales y las convenciones, según cada caso, son capaces de conducir el comportamiento de actores internacionales, moldeando expectativas y comportamientos en su ámbito de acción (Deudney e Ikenberry, 2999: 186). Siendo así, este tipo de estructuras e instituciones “[...] coordinan el comportamiento Estatal para alcanzar ciertos resultados en áreas específicas, siendo también un fenómeno persuasivo. Sus patrones de acción generan expectativas y ayudan a la creación de normas que a su vez refuerzan las instituciones creadas" (Benítez y Rodríguez, 2011: 194).

Para analistas como Georg Sorensen, y en línea con los postulados cosmopolitas, en su sugerente libro Changes in Statehood, este tipo de ejercicios consagrados en normas internacionales pretenden "homologar" comportamiento en torno a propósitos comunes en temas clave, lo cual se consigue a través de incentivos, aunque en ocasiones también puede recurrirse a la coerción (Sorensen, 2001: 53).

Dichas normas, al activar "protocolos de actuación", mismos que “dominarán las futuras transacciones" (Holsti, 1967:494), pretenden disminuir la discrecionalidad presente en todo ejercicio de cooperación (puesto que no existe ninguna autoridad superior que obligue a los actores a colaborar), aumentando en consecuencia la predictibilidad del proceso y los resultados del ejercicio colaborativo en cuestión. Esto último es en particular relevante, dado que implica que dichas instituciones internacionales mediante sus respectivas normas, ya sean a través de organismos internacionales, regímenes y convenciones contribuyen al fomento de la gobernanza global.

\section{Consideraciones finales. Cosmopolitismo moderno, constructivismo y liberalismo institucional: diálogo teórico a tres bandas}

Como se indicó de manera sintética, la CID comprende un conglomerado de actividades de diversa naturaleza y motivaciones que mediante acciones colectivas entre actores de la sociedad internacional pretenden mejorar las condiciones de vida de las personas. Su instrumentación (básicamente desde 1945 a la fecha) conforma un crisol de experiencias y efectos de diversa índole los cuales son objeto de estudio desde distintos postulados teóricos, en razón de la naturaleza, perfil y experiencias concretas de su actuar.

En ese sentido, y como se argumenta en ese artículo, el ámbito solidario de sus acciones es propenso a ser explicado mediante los postulados cosmopolitas, constructivistas y liberales/institucionalistas. Estas fundamentaciones teóricas 
convergen en diversos planteamientos que giran en torno a la solidaridad y ética internacional, la colaboración incluyente y corresponsable de los Estados nacionales ante la erosión de su poder y las sociedades, el papel de las normas durante su ejercicio, el nuevo protagonismo de la sociedad civil global y, en definitiva, en el análisis de las acciones concertadas en pro de la generación de beneficios a favor de la humanidad que superan concepciones westfalianas clásicas. El giro ético, la justicia global, la superación de la razón de Estado, el gradual desmantelamiento de las fronteras tradicionales de la soberanía Estatal y los alcances humanos de la política internacional son los elementos constituyentes y coincidentes del diálogo que se propone entre el cosmopolitismo, el constructivismo y el liberalismo institucional. La apuesta teórica va en función de recalcar que la interdependencia hace corresponsables a los seres humanos de enfrentar y resolver los retos globales.

Sin embargo, y como se ha señalado en ocasiones previas (Prado y Ochoa, 2009), la CID enfrenta retos que atan su capacidad para activar procesos transformadores. De nueva cuenta, aunque no son teorías en absoluto convergentes entre sí, percibimos vasos comunicantes entre el cosmopolitismo, el constructivismo y el liberalismo institucional que explican dichas resistencias.

David Held y Kevin Young escriben al respecto criticando lo que se podría denominar una lectura incompleta de los tiempos actuales y la falta de visión de largo plazo por parte de los actores internacionales clásicos. Si bien Held y Young asumen que hay un sistema mundial de regulaciones y de mecanismos de coordinación y cooperación multilaterales, lo consideran débil (Held y Young, 2011: 605). En un trabajo en particular han destacado las debilidades de la gobernanza global en las regulaciones financieras internacionales, en la conducción de la seguridad internacional y de la protección del medio ambiente (Held y Young, 2011: 605-635). Sus conclusiones, como se verá en seguida, coinciden plenamente con las aseveraciones acerca de las debilidades y retos de la CID contemporánea.

Held y Young parten de la afirmación de que las regulaciones internacionales funcionan limitadamente porque las instituciones requieren mejoras en función del sistema internacional multilateral que ya no opera sobre la base unilateral. Ambos autores señalan dos problemas relevantes: 1) el problema de la capacidad sostiene que "las capacidades institucionales existentes no son las adecuadas para lidiar con la naturaleza global de los riesgos ni con la presencia de externalidades negativas: en muchos sentidos, las instituciones actuales son incompetentes para alcanzar los objetivos necesarios; 2) el problema de la responsabilidad plantea que el sistema de regulaciones internacionales (el financiero, el de seguridad y el ambiental) es tan amplio y variado que "la propagación de los riesgos, al igual que la de los costos para soportar su realización, no son proporcionales con el número de participantes involucrados en su gestión (Held y Young, 2011: 606). 
Una muestra de esto es el déficit democrático y de eficacia de los principales organismos multilaterales heredados del siglo XX (ONU, Banco Mundial, FMI, OMC), los cuales carecen de estructuras adecuadas para hacer valer la voz y promover los intereses de la mayoría de la población mundial. De ahí el reclamo cosmopolita referido en párrafos previos, el sentido de la urgente necesidad de rediseñar este tipo de organismos, estructuras e instituciones de las cuales depende en buena medida la agenda global del desarrollo, señalamiento que los liberales institucionalistas respaldan de manera enfática.

En concordancia con la debilidad de la gobernanza global planteada por Held y Young, desde la perspectiva del liberalismo institucional también se detectan importantes elementos de oportunidad que impiden un alcance más amplio e incluyente de la CID. Las más destacadas son: 1) una endeble voluntad política por parte de los países (particularmente los más poderosos) para promover compromisos globales en favor de la reducción de la inequidad; 2) la selectividad temática en función de los intereses de los actores poderosos para diseñar la agenda global de la CID; 3) la selectividad geográfica atendiendo a los mismos esquemas que la selectividad temática; 4) el liderazgo restringido y, por ende, no representativo que guían las prioridades de la CID (fundamentalmente el G-7, G-20 y la OCDE); 5) el déficit democrático de los mecanismos de gobernanza global (G-20, Consejo de Seguridad de Naciones Unidas); 6) la incorporación de nuevos actores con sus propios esquemas y agendas de CID no siempre convergentes entre sí; 7) las promesas no vinculantes que distorsionan el tipo de ayuda ofrecida y la que se otorga; 8) el alcance limitado de la CID ante los viejos y nuevos dilemas de la inequidad global; 9) la vulnerabilidad de la CID ante coyunturas globales, regionales y locales o ante el cambio de prioridades de los actores donantes (Prado, 2015: 213-219).

Por lo tanto, se aprecia que no existe, a pesar de los riesgos compartidos, un sistema de regulaciones internacionales eficiente ni un sistema de cooperación internacional cohesionado. Todo esto es evidencia empírica que repercute en contra de la posibilidad de construir una verdadera gobernanza global que supere en paradigma estado céntrico del sistema internacional que plantean sobre el papel cosmopolitas, constructivistas y liberales institucionalistas.

Es claro que la frontera entre intereses nacionales y globales es uno de los asuntos más álgidos de la CID, dado que su actuar debe converger a favor de ambos propósitos, aunque en la mayoría de los casos es el interés nacional del proveedor (incluso países en desarrollo) el principal argumento -y motivaciónpor parte de varios gobiernos para realizar este tipo de actos (Quadir, 2013: 328). En ese sentido, de nueva cuenta el cosmopolitismo acierta en señalar el hecho de las dificultades que enfrentan procesos a favor del desarrollo global en razón a las inercias nacionalistas que en varios casos impiden ejercicios cooperativos de mayor envergadura que trasciendan a propósitos locales, 
patrióticos o nacionalistas de corto alcance. El que desde la guerra fría hasta hoy en día cerca del 70\% del promedio de la Asistencia Oficial para el Desarrollo (recursos de los principales donantes/países desarrollados para financiar sus respectivas actividades de CID) y de la cooperación Sur-Sur se otorguen por la vía bilateral, mientras que el 30\% de recursos restantes se dediquen a ejercicios colaborativos del orden regional, triangular y multilateral avala el postulado de Held respecto a "la paradoja de nuestro tiempo", en donde los intereses políticos económicos y de seguridad continúan primando en la agenda internacional por encima de propósitos en pro de un desarrollo más equitativo a escala mundial.

Por su parte, los argumentos cosmopolitas con respecto a la tradición liberal y ética con la que las relaciones internacionales son compartidos por el constructivismo - tal y como se señaló e líneas atrás, en donde la obligación moral de personas y colectividades para colaborar con entidades humanas que trascienden a las fronteras- constituyen el elemento de mayor empatía entre estas dos perspectivas teóricas.

A su vez, los vínculos entre el cosmopolitismo con el liberalismo institucional llaman la atención, en particular respecto a que el primero resalta la existencia de instituciones que pretenden conducir comportamiento de los actores internacionales a favor de propósitos colectivos; tal y como el segundo planteamiento estipula en sus preceptos.

En este sentido la sintonía entre cosmopolitismo y el neoliberalismo interdependiente es clara dado a que ambos planteamientos valoran el papel de la interconexión de una gama cada vez más amplia de actores del sistema internacional; procesos multinivel de transnacionalización que generan crecientes experiencias de cooperación internacional que superan la lógica Estatal y las fronteras territoriales tradicionales. En este sentido los postulados cosmopolitas dedicados a la necesidad de que el mundo gire en torno a instituciones que regulen el comportamiento humano marca un punto de inflexión con el planteamiento central del liberalismo institucional.

En esta línea argumentativa, ambos preceptos decantan su atención en la gobernanza global, la cual más allá de un gobierno formal, estipula la conformación de normas y reglas formales e informales que mediante múltiples ejercicios multinivel direccionan los asuntos públicos de interés colectivo. De ahí que el concepto de gobernanza de la CID, entendido como "conjunto de propósitos, regulaciones y procesos formales e informales establecidos mediante distintas instituciones internacionales a través de determinados actores que de manera permanente pretenden ser instrumentados y homologados a escala global, cuyo propósito es promover el bienestar humano" (Prado, 2016: 47) constituye en todo sentido un planteamiento afín al cosmopolitismo institucional. 
Como corolario, y a la luz de los razonamientos y argumentaciones aquí esgrimidos, es evidente que el cosmopolitismo, constructivismo y liberalismo institucional, más allá de sus diferencias, debido a sus similitudes en cuanto a la ontología de los fenómenos que analizan, celebran un diálogo teórico entre sí, que puede contribuir a analizar fenómenos concretos en donde la CID es un ejemplo representativo de tal situación.

Seguramente la divergencia más notoria entre estas tres categorías analíticas constituye que el cosmopolitismo, más allá de una teoría para explicar determinados sucesos (como lo hacen el constructivismo y el liberalismo institucional), pretende constituirse en un argumento ético-moral, a favor del cambio y transformaciones estructurales del orden internacional. Por tanto, podría decirse que el cosmopolitismo es más normativo que las otras dos teorías aquí revisadas. 


\section{Referencias bibliográficas:}

Barbé, Esther (2007), Relaciones Internacionales, Tecnos, Madrid.

Beate, Jahn (Ed.) (2006). Classical Theory in International Relations. Cambridge University Press: Cambridge.

Beck, U. (2005). La mirada cosmopolita o la guerra es la paz. Barcelon, Paidós. Beck, Ulrich (2006), La sociedad del riesgo. Hacia una nueva modernidad, Madrid, Paidós.

Benítez, Raúl y Rodríguez, Carlos (2011), Inseguridad, instituciones e integración subregional. Los dilemas de la Iniciativa Mérida, en Chanona Burguete, Alejandro, et. al (Coords.), Los regionalismos frente a la agenda de seguridad internacional, UNAM, México.

Brassetta, James \& Bulleyb, Dan (2007), "Ethics in World Politics: Cosmopolitanism and Beyond?", en International Politics, Vol 44, pp. $1-18$.

Cooper, Robert (2003), The Breaking of Nations, New York, Groove Press.

Czempiel, Ernst-Otto and Rosenau, James (eds) (1992), Governance without Government: Order and Change in World Politics, Cambridge, Cambridge University Press.

Deudney, Daniel e Ikenberry, John (1999), "The nature and sources of liberal international order", Review of International Studies, no. 25.

Figueroa Fischer, Bruno (2014) "El nuevo rostro de la cooperación internacional mexicana", Revista Mexicana de Política Exterior, no. 102, Instituto Matías Romero, México.

Griffiths, Martin, Terry O'Callaghan and Steven C. Roach (2002), International Relations. The Key Concepts. Second Edition, London \& New York, Routledge.

Griffiths, Martin, Roach Steven C., \& Solomon, M. Scot (2009), Fifty Key Thinkers in International Relations (Second Edition), London \& New York, Routledge.

Heater, Derek (1996), World Citizenship and Government. Cosmopolitan Ideas in the History of Western Political Thought, New York, Palgrave MacMillan,

Held, David (1995), Democracy and the Global Order. From the Modern State to Cosmopolitan Governance, Stanford, California, Stanford University Press.

Held, David (2005), "Los principios del orden cosmopolita", en Anales de la Cátedra Francisco Suárez, Vol. 39, pp. 133-151.

Held, David y Young, Kevin (2011), "Más allá de la crisis financiera: conceptualización de las debilidades de la gobernanza global", en Foro Internacional, Vol. LI, No. 4, octubre-diciembre, pp. 605-635. 
Held, David (2012), Cosmopolitismo. Ideales y Realidades. Madrid: Alianza Editorial.

Jackson, Robert y Sorensen, Georg (2010), International Relations. Theories and Approaches, Oxford University Press, Oxford.

Holsti, K, J (1967), International Politics. A Framework for Analysis, Eaglewood Cliffs, New Jersey.

Hook, W. Steven (1995), National Interest and Foreign Aid, Lynne Rieener, Washington.

Karns, Margaret y Mingts, Karen (2010), International Organizations. The Politics and Processes of Global Governance, Lynne Rienner Publishers, London.

Jackson, Robert y Sorensen, Georg (2010), International Relations. Theories and Approaches, Oxford University Press, Oxford.

Kendall, Gavin, Woodward, Ian \& Skrbis, Zlato (2009), The Sociology of Cosmopolitanism. Globalization, Identity, Culture and Government, New York, Palgrave MacMillan.

Lumsdaine, David H. (1993), Moral Vision in International Politics. The Foreign Aide Regime 1949-1989, , Princeton, University Press, Princeton.

Keohane Robert O (1984), After Hegemony. Cooperation and Discord in the World Political Economy, Princeton University Press, 1984

Krasner, Stephen D. (Ed) (1983), International Regimes, Ithaca, Coronell University Press.

Kornprobst, Markus (2009), International Relations as Rhetorical Discipline:

Toward (Re)Newing Horizons, en International Studies Review, No. 11, pp. 87-108

Linklater, Andrew and Suganami, Hidemi (2006). The English School of International Relations. A Contemporary Reassessment, Cambridge University Press, Cambridge.

Leibfried, Stephan and Zürn, Michael (eds) (2005), Transformations of the State? Cambridge, Cambridge University Press.

López-Vallejo, Marcela (2016), "Gobernanza global”, en Schiavon, J. A., Ortega, A., López-Vallejo, M., Velázquez, R. (editores), Teorías de relaciones internacionales en el siglo XXI. Interpretaciones criticas desde México, CIDE/AMEI/BUAP/COLSAN/UABC/UANL/UPAEP, pp. 473491.

McGrw, Anthony (2004), "Cosmopolitanism and Global Justice", en Ritsumeikan Annual Review of International Studies, Vol. 3 pp. 1-17.

Millán, Carla (2014) "Cosmopolitización del desarrollo y coherencia de políticas: nuevos desafíos para la agenda post 2015", en Investigación y Desarrollo, Vol. 22, No. 1, pp. 168-186. 
Pierre, Jon (ed.) (2000), Debating Governance: Authority, Steering and Democracy, Oxford, Oxford University.

Prado Lallande y Ochoa Bilbao, Luis (2009), "El sistema de cooperación internacional para el desarrollo: frente a la securitización y la crisis económica global", Revista de Relaciones Internacionales de la UNAM, no. 105, Facultad de Ciencias Políticas y Sociales - -Universidad Nacional Autónoma de México, México.

Prado Lallande, Juan Pablo (2015), La cooperación internacional para el desarrollo. Acciones, desafios, tendencias, Madrid: La Catarata.

(2016), "La gobernanza de la cooperación Sur-Sur", en Soares de Lima, María Regina, (et. al)., Cooperación Sur-Sur, política exterior y modelos de desarrollo en América Latina, CLACSO, Buenos Aires.

(2016b), "El Neoliberalismo Institucional", en Schiavón, Jorge, et al., Teorías de las Relaciones Internacionales en el siglo XXI. Interpretaciones críticas desde México, CIDE, BUAP, UABJ, México.

Quadir, Fahimul (2013), "Rising Donors and the New Narrative of South South Cooperation: what prospects for changing the landscape of development assistance programmes?" Third World Qualterly, vol. 32, no. 2.

Riddell, Roger, C. (1987), Foreign Aid Reconsidered, Jamed Curie and Overseas development Insitute.

Rubin, Alfred (1980), "Review on Political Theory and International Relations", en The University of Chicago Law Review, pp. 403-414.

Sand, Inger-Johanne (2004), "Polycontextuality as an Alternative to Constitutionalism", in C. Joerges, I-J. Sand and G. Teubner (eds), Transnational Governance and Constitutionalism, Oxford, Hart Publishing, pp. 41-66.

Slaughter, Anne-Marie (2004), A New World Order, Princeton, New Jersey, Princeton University Press.

Sorensen, Georg (2001), Changes in Statehood. The Transformation of International Relations, Palgrave, London.

Sutch, Peter and Elias, Juanita (2007), International Relations: The basics, London \& New York, Routledge.

Tinnevelt, Ronald \& Verschraegen (eds.) (2006), Between Cosmopolitan Ideals and State Sovereignty. Studies in Global Justice, New York, Palgrave MacMillan.

Vernon, Richard (2010), Cosmopolitan Regard. Political Membership and Global Justice, Cambridge, U.K., Cambridge University Press.

Wallerstein, Immanuel (coord.) (2007), Abrir las ciencias sociales, México, Siglo XXI/UNAM. 
Weiss, Thomas G \& Wilkinson, Rorden (2014), “Global Governance al rescate: ¿salvando las relaciones internacionales?” en Foro Internacional, Vol. LIV, 1 (215) Enero-Marzo, pp. 76-105.

Wendt, Alexander(1995), "Constructing International Politics," en International Security, Center for Science nad International Affairs, Harvard University, MIT Press, Summer, Vol. 20, no. 1.

Young Oran R, International Cooperation. Building Regimes for Natural Resources and the Environment, Cornell University Press, London, 1989. 
